\title{
Plain and oscillatory solitons of the cubic complex Ginzburg-Landau equation with nonlinear gradient terms
}

\author{
M. Facão* \\ Departamento de Física, Universidade de Aveiro and I3N \\ Campus Universitário de Santiago, 3810-193 Aveiro, Portugal \\ M. I. Carvalho ${ }^{\dagger}$ \\ DEEC/FEUP and INESCPorto, Universidade do Porto, \\ Rua Dr. Roberto Frias, 4200-465 Porto, Portugal
}

\begin{abstract}
In this work, we present parameter regions for the existence of stable plain solitons of the cubic complex Ginzburg-Landau equation (CGLE) with higher order terms associated with a fourth order expansion: using a perturbation approach around the nonlinear Schrödinger equation soliton and a full numerical analysis that solves an ordinary differential equation for the soliton profiles and uses the Evans method in the search for unstable eigenvalues, we have found that the minimum equation allowing these stable solitons is the cubic CGLE plus a term known in optics as Raman delayed response which is responsible for the red shift of the spectrum. The other favorable term for the occurrence of stable solitons is a term that represents the increase of nonlinear gain with higher frequencies. At the stability boundary for higher values of the nonlinear gain, a bifurcation occurs giving rise to stable oscillatory solitons. These oscillations can have very high amplitudes, with the pulse energy changing more than two orders of magnitude in a period, and can even exhibit more complex dynamics such as period doubling.
\end{abstract}

\footnotetext{
*mfacao@ua.pt

$\dagger$ mines@fe.up.pt
} 


\section{INTRODUCTION}

In the optics context, the complex Ginzburg-Landau equation (CGLE) has been applied to describe pulse pulse propagation in fibers with linear and nonlinear gain and spectral filtering [1], pulse generation in fiber lasers with additive pulse mode-locking [2] or pulse propagation in hollow core photonic crystal fibers filled with resonant gases [3]. The CGLE can take the cubic or quintic forms, both of them supporting dissipative stationary solitons that result from a balance between dispersion and nonlinearity, and between gain and loss. In the cubic CGLE, these solitons are stable in a parameter region where the background is unstable, thus preventing the existence of stable stationary solutions. However, it has been recently shown that the inclusion in the CGLE of a term that, in optics, models the delayed Raman scattering, allows the existence of stable solutions in a parameter region where the background is also stable [4]. Note that this higher order term has also been associated with the stabilization of erupting solitons of the quintic CGLE [5-7].

The CGLE has been associated with extremely rich dynamical behaviors, of which stationary solutions are just one example. On the opposite side we find chaotic behavior and, somewhere in the middle, the oscillatory behavior typical of pulsating solitons [8, 9]. This oscillatory behavior has only been predicted for the quintic form of the CGLE, either with $[10,11]$ or without higher-order terms $[8,12,13]$, and, recently, has become the focus of renewed interest due to the unusually high peak amplitude oscillations that can be obtained [14].

Generalizations of the cubic CGLE including terms associated with complex coefficients of group velocity, third order dispersion, Raman scattering, self-steepening have been proposed in the context of pulse propagation in resonant media [3, 15]. The terms of Raman scattering and self-steepening are also known in fluid dynamics as the nonlinear gradient terms and their effect on the quintic CGLE have been already studied $[8,10,16]$, but here we study them in the cubic CGLE. A preliminary study of the effect of some of these terms has already been performed [4] and it was shown that, among others, they can affect the pulse peak amplitude and propagation velocity. Here, we expand on those results by using both perturbation analysis and numerical methods to obtain the plain soliton characteristics. Furthermore, these two approaches are also used to investigate how these higher order terms affect the region of existence and stability of these solitons. Direct numerical integration is 
also used to confirm the results obtained and to establish the basin of attraction of these stationary pulses. Note that the term plain solitons is usually used in the terminology of stable solutions of the CGLE to designate the one-hump constant amplitude profile solution. Finally, our results indicate that other kind of solutions are also possible in the CGLE of the cubic type in a parameter region that is beyond the stability region of plain solitons. They exhibit a rich behaviour of periodic or aperiodic oscillations of shape and peak amplitude. To the best of our knowledge, this is the first time a behavior of this sort is predicted for this form of the CGLE.

Section II of this article addresses the existence of stable traveling solutions of the cubic CGLE with higher-order terms and is divided into two parts: the first with results from a perturbation approach and the second one dedicated to numerical methods. The oscillatory solutions are presented in Section III, and in section IV we summarize the main results of this work.

\section{EXISTENCE AND STABILITY OF PLAIN SOLITONS}

Consider the evolution equation

$$
i q_{Z}-\frac{D}{2} q_{T T}+s|q|^{2} q=i \delta^{\prime} q+\xi^{\prime} q_{T}+i \beta q_{T T}+i d_{3}^{\prime} q_{T T T}+i \epsilon|q|^{2} q+R^{\prime}\left(|q|^{2}\right)_{T} q-i S^{\prime}\left(|q|^{2} q\right)_{T}
$$

where, in the optical context, $q$ is the normalized envelope of the optical field, and $Z$ and $T$ are the normalized propagation distance and retarded time, respectively. The parameters in this equation are all normalized versions of the actual parameters. The parameters $D$ and $s$ may only take the values $\pm 1, D=1$ if the group velocity dispersion (GVD) is normal and -1 if GVD is anomalous and $s=1$ or $s=-1$ for positive or negative Kerr effect, respectively. $\delta^{\prime}$ stands for linear gain/loss, gain if $\delta^{\prime}>0$ and loss otherwise, $\xi^{\prime}$ represents a linear frequency dependent gain/loss, it will cause loss in one side of the spectrum and gain in the other side, $\beta(>0)$ is a quadratic frequency dependent loss, easily recognized as a spectral filtering and $\epsilon$ is responsible for nonlinear gain/loss, gain if $\epsilon>0$ and loss otherwise. $S^{\prime}=S_{r}^{\prime}+i S_{i}^{\prime}$ is the parameter associated with the term that models the frequency dependency of the cubic terms, its real part is associated with the so called self-steepening and represents the dependency of the Kerr term with frequency (it is conservative) and the imaginary part is associated with the frequency dependency of the nonlinear gain/loss which is dissipative. 
The parameter $R^{\prime}=R_{r}^{\prime}+i R_{i}^{\prime}$ represents a delayed Kerr and nonlinear gain/loss response. Both components of this term are conservative for signals that are localized in time. Finally, $d_{3}^{\prime}=d_{3 r}^{\prime}+i d_{3 i}^{\prime}$ corresponds to third order dispersion $\left(d_{3 r}^{\prime}\right)$ and to cubic frequency dependent gain/loss $\left(d_{3 i}^{\prime}\right)$. Note that this equation is the cubic complex Ginzburg-Landau equation (CGLE) with higher-order (HOT) terms added in the form of $\xi, R, S$ and $d_{3}$.

We may reduce the number of parameters of equation (1) using the following change of variables

$$
p=\frac{q}{a}, \quad \zeta=a^{2} Z, \quad \text { and } \quad \tau=a T,
$$

with which the evolution equation reads

$$
i p_{\zeta}-\frac{D}{2} p_{\tau \tau}+s|p|^{2} p=i \delta p+\xi p_{\tau}+i \beta p_{\tau \tau}+i d_{3} p_{\tau \tau \tau}+i \epsilon|p|^{2} p+R\left(|p|^{2}\right)_{\tau} p-i S\left(|p|^{2} p\right)_{\tau}
$$

In the above equation $\delta=\delta^{\prime} / a^{2}, d_{3}=d_{3}^{\prime} a, \xi=\xi^{\prime} / a, R=a R^{\prime}$ and $S=a S^{\prime}$ and if we choose $a^{2}=-\delta^{\prime}, \delta=-1$. Note that we will be interested only in the case $\delta^{\prime}<0$.

\section{A. Perturbation approach}

We will restrict our analysis here to the case $D s=-1$, such that the left-hand side of (1) becomes the focusing nonlinear Schrödinger equation (NLS). Then, we proceed by applying standard inverse scattering method perturbation analysis $[17,18]$ considering the fundamental soliton of the NLS, namely, $p(\tau, \zeta)=\eta(\zeta) \operatorname{sech}\left[\eta(\zeta)\left(\tau-\tau_{0}(\zeta)\right)\right] \exp \left[i b(\zeta)\left(\tau-\tau_{0}(\zeta)\right)+\right.$ $i \phi(\zeta)]$, and obtain the following equations for $\eta$ and $b$

$$
\begin{gathered}
\frac{d \eta}{d \zeta}=2 \eta\left[-1+\xi b-\beta\left(b^{2}+\frac{\eta^{2}}{3}\right)+\frac{2}{3} \eta^{2}\left(\epsilon+S_{i} b\right)+d_{3 i} b\left(\eta^{2}+b^{2}\right)\right] \\
\frac{d b}{d \zeta}=\frac{2}{3} \eta^{2}\left[\xi-2 \beta b+\frac{2}{5} \eta^{2}\left(2 R_{r}+3 S_{i}\right)+3 d_{3 i}\left(b^{2}+\frac{7}{15} \eta^{2}\right)\right]
\end{gathered}
$$

and also $\frac{d \tau_{0}}{d \zeta}=-D b+\eta^{2}\left(S_{r}-\frac{2}{3} R_{i}\right)+d_{3 r}\left(\eta^{2}+3 b^{2}\right)$ and $\frac{d \phi}{d \zeta}=\frac{D}{6}\left(\eta^{2}-3 b^{2}\right)+\frac{2}{3} s \eta^{2}+$ $\frac{1}{3} b \eta^{2}\left(S_{r}-2 R_{i}-b^{2}\right)$.

Since the evolution equations for the perturbed soliton parameters only involve $\eta$ and $b$, we only have to take into account equations (2) and (3) in order to find the stationary amplitude and frequency. Thus, in practice, we are approximating the behaviour of the infinite dimensional system governed by equation (1) by a two-dimensional system. Furthermore, as 
it will be shown later in this section, in order to ensure background stability we must have $\beta>0$ and $\frac{\xi^{2}}{4 \beta}<1$, and the $d_{3}$ coefficient must be real. In this case $\left(d_{3 i}=0\right)$, the equilibrium nontrivial amplitude and frequency are given by

$$
\begin{gathered}
b_{e}=\frac{1}{\beta}\left(\frac{\xi}{2}+\frac{1}{5}\left(2 R_{r}+3 S_{i}\right) \eta_{e}^{2}\right) \\
-\frac{\left(6 R_{r}-S_{i}\right)\left(2 R_{r}+3 S_{i}\right)}{75 \beta} \eta_{e}^{4}+\frac{1}{3}\left(2 \epsilon-\beta+\frac{S_{i} \xi}{\beta}\right) \eta_{e}^{2}-1+\frac{\xi^{2}}{4 \beta}=0 .
\end{gathered}
$$

Note that these equations are more general than the ones presented in [4], which were obtained assuming that $\xi=0, S=0, d_{3}=0$ and $R_{i}=0$. However, similarly to what happens when these coefficients are not present, our analysis indicates that three different types of parameter regions can be identified: one where no stationary solution is allowed, one with only one solution, and a third one with two different solutions that we name as low amplitude and high amplitude solutions. Furthermore, only the high-amplitude solution in the two solutions regime can be stable. In the following we present the details leading to these conclusions.

A nontrivial equilibrium solution requires $\eta_{e}^{2}$, given by (5), to be real and positive. Since we are considering $\beta>0$ and $\frac{\xi^{2}}{4 \beta}<1$, we can conclude that

1. (region 1) Two positive solutions for $\eta_{e}$ are allowed if $R_{r} \neq 0,-\frac{2}{3}<\frac{S i}{R_{r}}<6$ and $\epsilon>\epsilon_{\min }$ with

$$
\epsilon_{\min }=\frac{1}{2} \beta-\frac{S_{i} \xi}{2 \beta}+\frac{3}{5} \sqrt{\left(1-\frac{\xi^{2}}{4 \beta}\right) \frac{\left(6 R_{r}-S_{i}\right)\left(2 R_{r}+3 S_{i}\right)}{3 \beta}} .
$$

2. Only one positive solution for $\eta_{e}$ is possible in the following situations:

(a) (region 2) $R_{r} \neq 0,-\frac{2}{3}<\frac{S i}{R_{r}}<6$ and $\epsilon=\epsilon_{\min }$,

(b) (region 3) $S_{i}=6 R_{r}$ or $S_{i}=-\frac{2}{3} R_{r}$ and $\epsilon>\epsilon_{\min } \equiv \frac{\beta}{2}-\frac{S_{i} \xi}{2 \beta}$,

(c) (region 4) $S_{i} \neq 0$ and $-\frac{3}{2}<\frac{R_{r}}{S_{i}}<\frac{1}{6}$ for every $\epsilon$.

3. (region 5) Finally, no solution is predicted in all other cases.

Some valuable insights regarding the stability of system (1) can also be obtained by addressing the stability of these stationary points of the 2-dimensional system (the perturbed solitons). This can be investigated by looking at the eigenvalues of the $2 \times 2$ jacobian matrix 
associated with the right-hand side of equations (2) and (3), calculated at the stationary points. In particular, the $2 \mathrm{D}$ system will be stable as long as the real part of these eigenvalues is negative. In the present case and using again $\beta>0$, this translates to two conditions

$$
\begin{gathered}
\frac{1}{75 \beta}\left(2 R_{r}+3 S_{i}\right)\left(6 R_{r}-S_{i}\right) \eta_{e}^{4}>1-\frac{\xi^{2}}{4 \beta}, \\
\frac{10 S_{i}\left(2 R_{r}+3 S_{i}\right)}{75 \beta} \eta_{e}^{2}+\frac{2}{3}(\epsilon-\beta)+\frac{S_{i} \xi}{3 \beta}<0 .
\end{gathered}
$$

The first condition is only met by the high amplitude solution of the two solution regime, implying that only that solution can possibly be stable. By the perturbation approach, it will be stable if the other condition is also satisfied.

We may now address the full scenario of traveling pulse solutions to equation (1), as given by the NLSE perturbation approach. The low amplitude solutions are the continuation of the chirped solutions of the cubic CGLE, that there, and for $\frac{\xi^{2}}{4 \beta}<1$, only exist above $\epsilon_{\min }^{\mathrm{CGLE}}=\beta / 2[3,19]$. As we introduce the Raman term and if $-\frac{2}{3}<\frac{S_{i}}{R_{r}}<6$, we gain a new solution, higher in amplitude, that exists on the same parameter region as the low amplitude solution. In the same conditions but at $\epsilon_{\min }$, the two solutions coincide, such that, there is only one solution. If otherwise, $S_{i}=6 R_{r}$ or $S_{i}=-\frac{2}{3} R_{r}$, we have a situation identical to the one obtained with the cubic CGLE, namely, a single solution above $\epsilon_{\min }$. In a third case, if $S_{i} \neq 0$ and $-\frac{3}{2}<\frac{R_{r}}{S_{i}}<\frac{1}{6}$, there is only the low amplitude solution but for all $\epsilon$. Nevertheless, from all of these, the only stable pulse is the high amplitude solution allowed when $R_{r} \neq 0$ in region 1 , but for $\epsilon$ that obeys condition (6). In some cases, as is the case $S_{i}=0$, the condition (6) reduces to $\epsilon<\epsilon_{\max }=\beta$ and the existence of a finite region with stable solitons implies that $\epsilon_{\min }<\epsilon_{\max }$, which imposes an additional restriction on the coefficients of (1), they will exist as long as $\left|R_{r}\right|<\frac{5}{12} \beta \sqrt{\frac{\beta}{1-\frac{\xi^{2}}{4 \beta}}}$. For $S_{i} \neq 0,(6)$ should be solved giving also an upper limit on $\epsilon$ or an interval, as we shall see in the examples below. We should remark that this perturbation analysis only predicts the existence of stable plain solitons for $R_{r} \neq 0$.

\section{B. Numerical approach}

The perturbation approach followed in the previous paragraphs would just be valid for small values of the parameters associated with the terms beyond the NLS. To further continue our search for solutions of $(1)$, we write $p(\zeta, \tau)$ as a traveling solution of the form 
$q(\zeta, \tau)=F(t) e^{i \theta(t)+i \omega \zeta}$, with $t=\tau-v \zeta$, where both $F$ and $\theta$ are real, and used a similar approach as used in [4] obtaining ordinary differential equations (ODEs) for $F$ and $\theta^{\prime}$ which were solved by a shooting method. Using the latter approach, we have scanned regions of the parameter space having always $R_{r} \neq 0$. In fact, we have found as doublets, a low amplitude solution and a high amplitude solution that is stable in a region smaller than the existence region, but we could also verify that, for parameters close to the region identified above as 4 , there is only one solution in a extended region of $\epsilon$. For the stability study, we have used an Evans function method [3] to evaluate the eigenvalues of the associated stability system, which reads

$$
L \mathbf{w}=\lambda \mathbf{w}, \quad \mathbf{w}=\left(\begin{array}{ll}
u & x
\end{array}\right)^{T}
$$

where

$$
\begin{gathered}
L=\left(\begin{array}{cc}
-i d_{3} \partial_{t t t}+\left(-\frac{D}{2}-i \beta\right) \partial_{t t}-(i v+\xi) \partial_{t} & B(t) \\
-\omega+i+A(t) & -i d_{3}^{*} \partial_{t t t}+\left(\frac{D}{2}-i \beta\right) \partial_{t t}-(i v-\xi) \partial_{t} \\
-B^{*}(t) & +\omega+i-A^{*}(t)
\end{array}\right) \\
A(t)=2(s-i \epsilon) F^{2}-R\left(3 F F^{\prime}+F^{2} \partial_{t}-i \theta^{\prime} F^{2}\right)+2 i S\left(2 F F^{\prime}+F^{2} \partial_{t}\right) \\
B(t)=\left[(s-i \epsilon) F^{2}-R\left(F F^{\prime}+F^{2} \partial_{t}+i \theta^{\prime} F^{2}\right)+S\left(2 i F F^{\prime}-2 \theta^{\prime} F^{2}+i F^{2} \partial_{t}\right)\right] \mathrm{e}^{2 i \theta} .
\end{gathered}
$$

The traveling pulses are linearly stable if $L$ has no eigenvalues $\lambda$ with negative imaginary part.

The location of the continuous spectrum may be estimated using the continuous spectrum of the limiting form of $L$ for $t \rightarrow \pm \infty$ which is obtained from $L$ putting $A(t)=B(t)=0$. The continuous spectrum of $L_{\infty}$ is given by

$$
\begin{gathered}
-d_{3} r^{3}+(D / 2+i \beta) r^{2}+(v-i \xi) r-\omega+i=\lambda, \\
-d_{3}^{*} r^{3}+(-D / 2+i \beta) r^{2}+(v+i \xi) r+\omega+i=\lambda,
\end{gathered}
$$

with $r$ being any real number. For the first curve, we have

$$
\begin{gathered}
\lambda_{r}=-d_{3 r} r^{3}+\frac{D}{2} r^{2}+v r-\omega \\
\lambda_{i}=-d_{3 i} r^{3}+\beta r^{2}-\xi r+1
\end{gathered}
$$




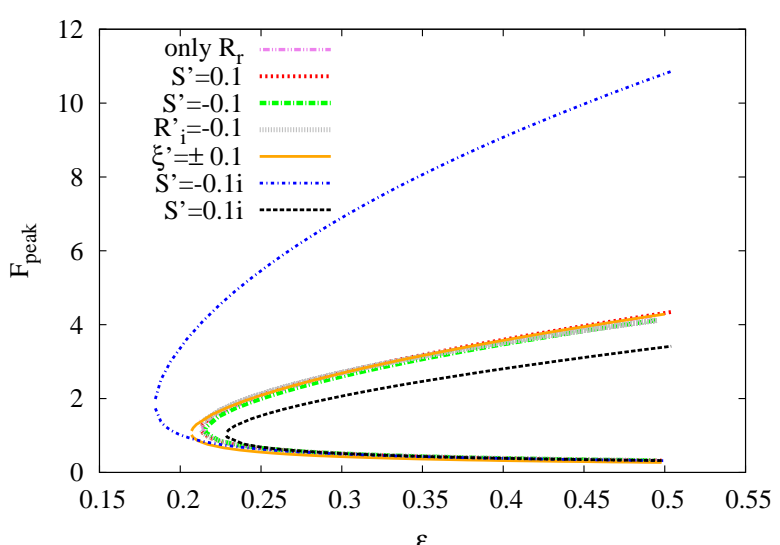

(a)

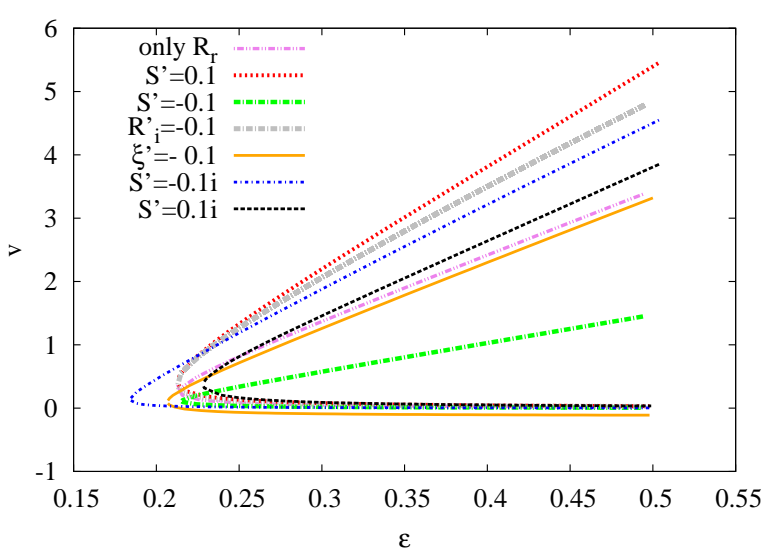

(b)

FIG. 1. Peak amplitude (a) and velocities (b) of the two branches of solutions for $\delta^{\prime}=-0.022$, $\beta=0.4, R^{\prime}=0.177$ and other nonzero parameters as indicated in the curve labels.

For a large enough $r$, the dominant term in the equation for $\lambda_{i}$ will be $-d_{3 i} r^{3}$. Since this term can take negative values regardless of the sign of $d_{3 i}$, we must require $d_{3 i}=0$ in order to allow for stable background. Moreover, since in this case $\lambda_{i}$ will attain a minimum value of $1-\frac{\xi^{2}}{4 \beta}$ if $\beta>0$, background stability is only possible when we also have $\frac{\xi^{2}}{4 \beta}<1$ and $\beta>0$. The continuous spectrum of $L$ itself is on or inside the above parabolas [20]. Hence, the continuous stability spectrum of these solutions is stable if $\xi^{2} / 4 \beta<1$, as we have referred in the last section.

First, we show the peak amplitude and velocities for the two branches of solutions, high and low amplitude solutions, for particular values of the parameters (see figure 1). The results were obtained by numerically solving the ODEs for $F$ and $\theta^{\prime}$ described above. The peak amplitudes for the different parameters are very similar, except for the high amplitude solutions whenever $S_{i}^{\prime}=-0.1$. However, the velocities show some dispersion. In the rest of this section, we will concentrate in the high amplitude solutions, studying the parameter region for their stability and showing some of the profiles. Thus, our results of existence and stability of the high-amplitude solution are presented in figures 2 and 3 in limited regions of $(\beta, \epsilon)$ for different choices $\xi$ and $S$ but having always $R_{r} \neq 0$, which by the perturbation analysis of section $\mathrm{A}$ is a necessary condition for the existence of stable solitons. In figure 4, we compare the amplitude and phase derivative profiles.

From our results, we learn that the existence and stability regions for relatively small $\beta$ and $\epsilon$ are well predicted by the perturbation approach. As $\beta$ increases, the tendency in 


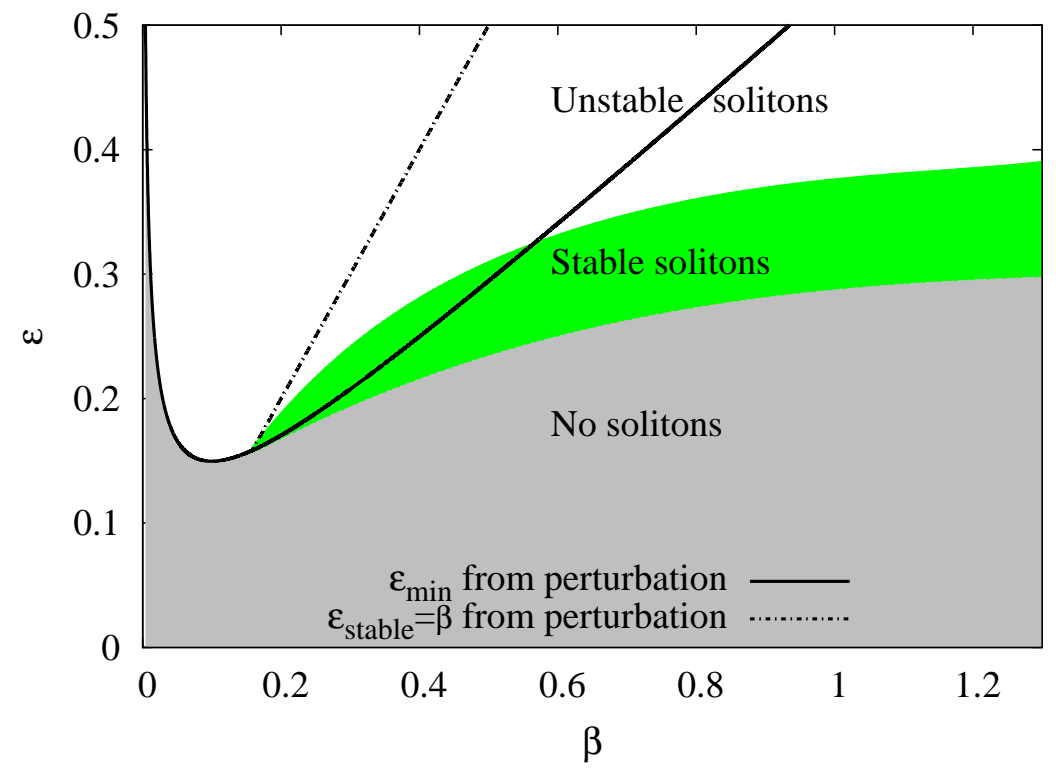

FIG. 2. Existence and stability for $\delta^{\prime}=-0.022, \xi=0, R^{\prime}=0.177$ and $S^{\prime}=0$. The three different regions are regions of absence of solitons, stable and unstable solitons as indicated in the figure.The two curves are the threshold for existence and stability as predicted by perturbation.

all graphs is an existence threshold lower than $\epsilon_{\min }$ and a stability threshold considerably lower than $\epsilon_{\max }$. However, this discrepancy with the perturbation results is not the only one. The major difference is in the phase profile. Since the perturbation results are based on the NLS soliton, the phase is assumed to vary linearly with time. However, starting with the work by Akhmediev in the 90s [19], it is known that there are analytical solutions of the cubic CGLE that have fixed amplitude and chirp such that the phase derivative has a tanh profile. For $\delta<0$ (needed for background stability), these solutions exist above a certain $\epsilon$, but are always unstable. As predicted by the perturbation approach and studied in [4], the introduction of $R_{r}$ creates a new solution with higher amplitude. The phase derivative of both solutions is not symmetrical but also tends to fixed values at the tails showing a rapid variation at the pulse center. The phase derivative of the high amplitude solutions may be observed in the dashed (black) lines of graphs in Fig. 4. Concerning the amplitude profiles, they are reasonably equal to sech, with a slight asymmetry, for smaller values of the parameters but they tend to be narrower than the sech as the parameter values increase.

Let us now follow the introduction of $S_{r}, R_{i}, \xi$ and $S_{i}$. The perturbation results predict that only $R_{r}, \xi$ and $S_{i}$ will affect the solution and its stability. However, the numerical results show different effects especially in some cases. If a positive $S_{r}$ does not introduce 
much variation on the solution profiles (amplitude and phase) relatively to the case of only nonzero $R_{r}$, a negative $S_{r}$ disturbs the phase, the phase derivative has more structure at the pulse site (fig. 4(a)) and the stability region increases in size (figures 3(a) and 3(b)).

On the other hand, the introduction of negative $R_{i}$ does not affect much the profiles and stability, but a positive $R_{i}$ introduces some phase derivative structure and a negative $R_{i}$ decreases the region of stability. We have noted some similarity between the results for negative $R_{i}$ and positive $S_{r}$ and vice-versa which might be anticipated since in the ODEs [4] the $R_{i}$ is always appearing in terms of the form $2 R_{i}-3 S_{r}$ which will be approximately the same for $R_{i}= \pm 0.15$ and $S_{r} \mp 0.1$. Nevertheless the two cases are not exactly the same since $S_{r}$ is also appearing in other forms in the ODEs.

The profiles for $\xi \neq 0$ are quite similar to the ones with only $R_{r}$ (fig. 4(c)). The amplitude profile is equal for $\xi$ negative and positive but the phase derivatives are displaced from each other. The existence and stability results obtained numerically agree with the one obtained with the perturbation approach for small $\beta$ (figure $3(\mathrm{~d})$ ). Note that the graph is shown only for $\beta>0.113$ which is the condition for stable background. The perturbation results for existence and stability do not distinguish the signal of $\xi$ since we are considering $S_{i}=0$ and thus $\xi$ only appears as $\xi^{2}$. The search of profiles using the ODEs and the Evans method confirm that fact.

Finally, the introduction of $S_{i}$ has a considerable influence on the results which was already anticipated by the perturbation approach. In the particular cases presented in figures $3(\mathrm{e})$ and $3(\mathrm{f})$, there is also an $\epsilon_{\max }$ obtained with (6) for $S_{i}^{\prime}=0.1$ but for $S_{i}^{\prime}=-0.1$ the stability region predicted by the perturbation approach is an interval for $\beta$ below 0.06 and only transforms to an upper limit beyond that $\beta$. The numerical results confirm this behavior for small $\beta$. In general, the stability region shrinks for positive $S_{i}$ and enlarges otherwise. These results are generally in agreement with the perturbation results but with displacement of the existence and stability regions as $\beta$ increases. The profiles are also quite different from the ones for $R_{r} \neq 0$, especially for negative $S_{i}$ for which the peak amplitude and rapid variation of the phase derivative at the pulse center are much higher than in the case of only $R_{r} \neq 0$. There is no fine structure in phase derivative at the pulse peak location.

Due to added numerical difficulties, in particular, the resulting ODEs being third order, we have not defined the existence and stability regions in the case $d_{3 r} \neq 0$. Thus, all the results that are shown below are for the case $d_{3 r}=0$. The case $d_{3 r} \neq 0$ will be considered 


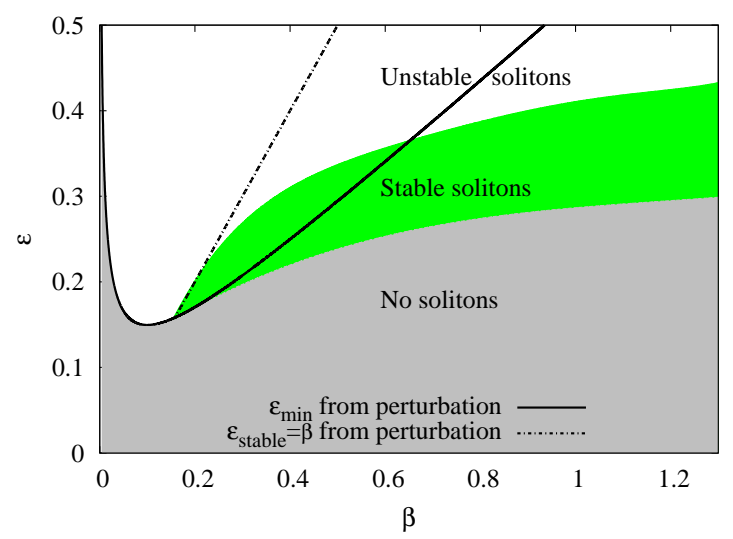

(a)

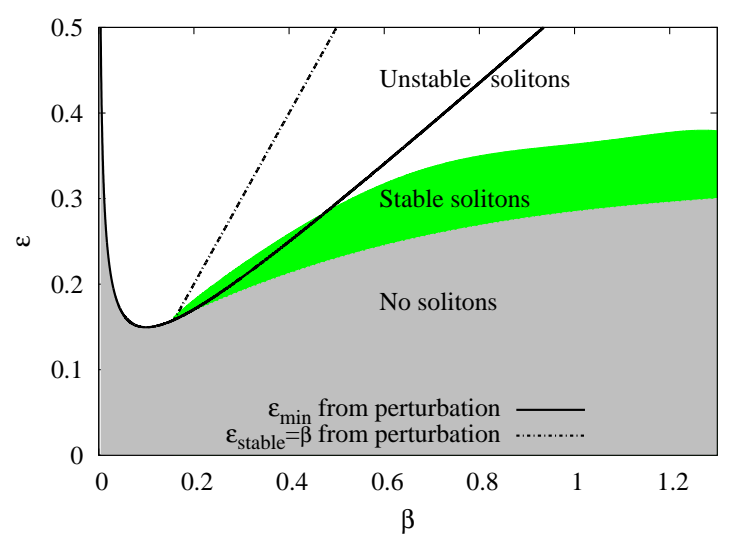

(c)

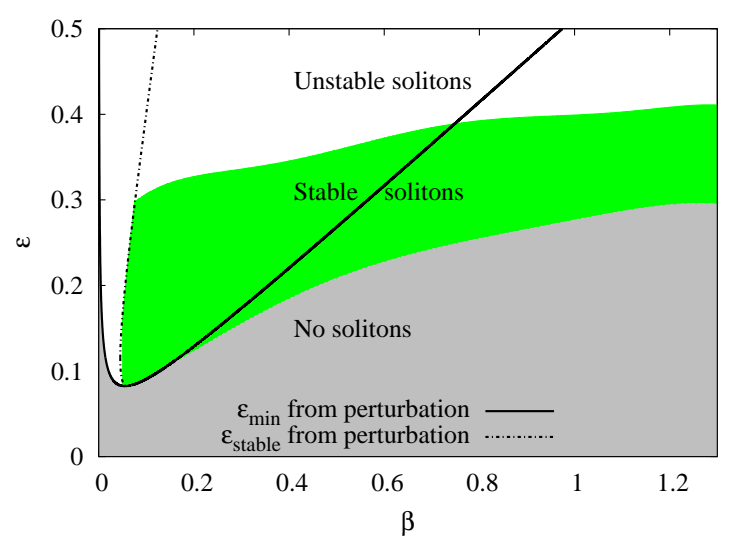

(e)

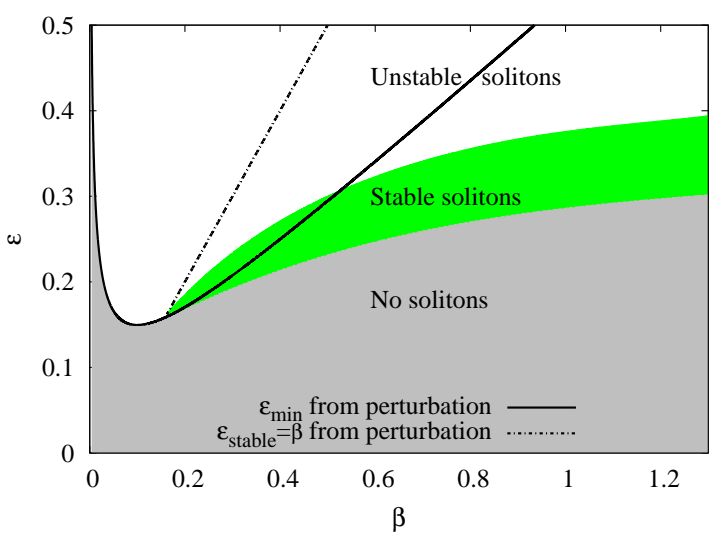

(b)

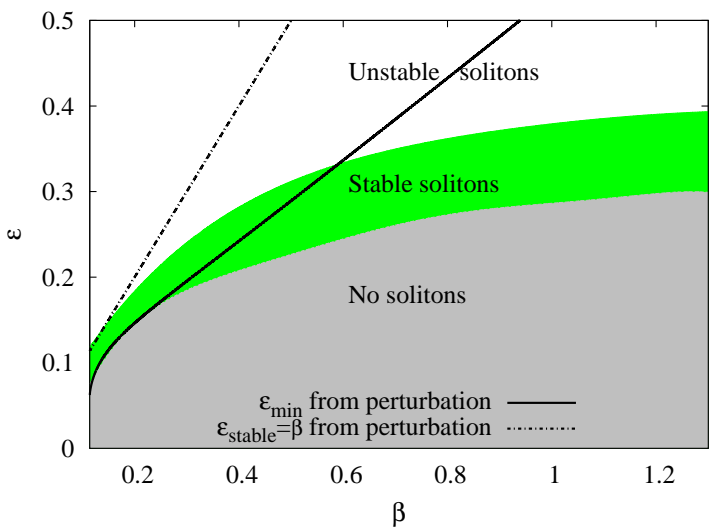

(d)

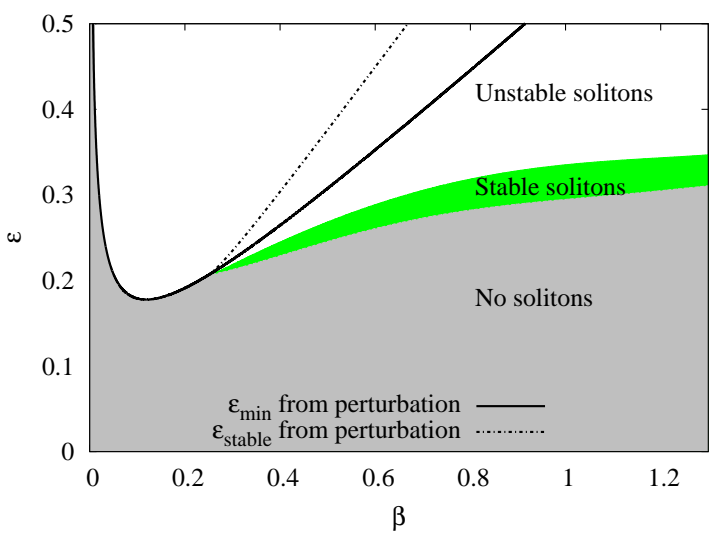

(f)

FIG. 3. Existence and stability for $\delta^{\prime}=-0.022, R^{\prime}=0.177$ and (a) $S^{\prime}=-0.1$, (b) $S^{\prime}=0.1$, (c) $R_{i}^{\prime}=0.14$, (d) $\xi^{\prime}= \pm 0.1$, (e) $S^{\prime}=-0.1 i$ and (f) $S^{\prime}=0.1 i$. The parameters that are not indicated are zero. The three different regions are regions of absence of solitons, stable and unstable solitons as indicated in the figures. The two curves are the threshold for existence and stability as predicted by perturbation. 

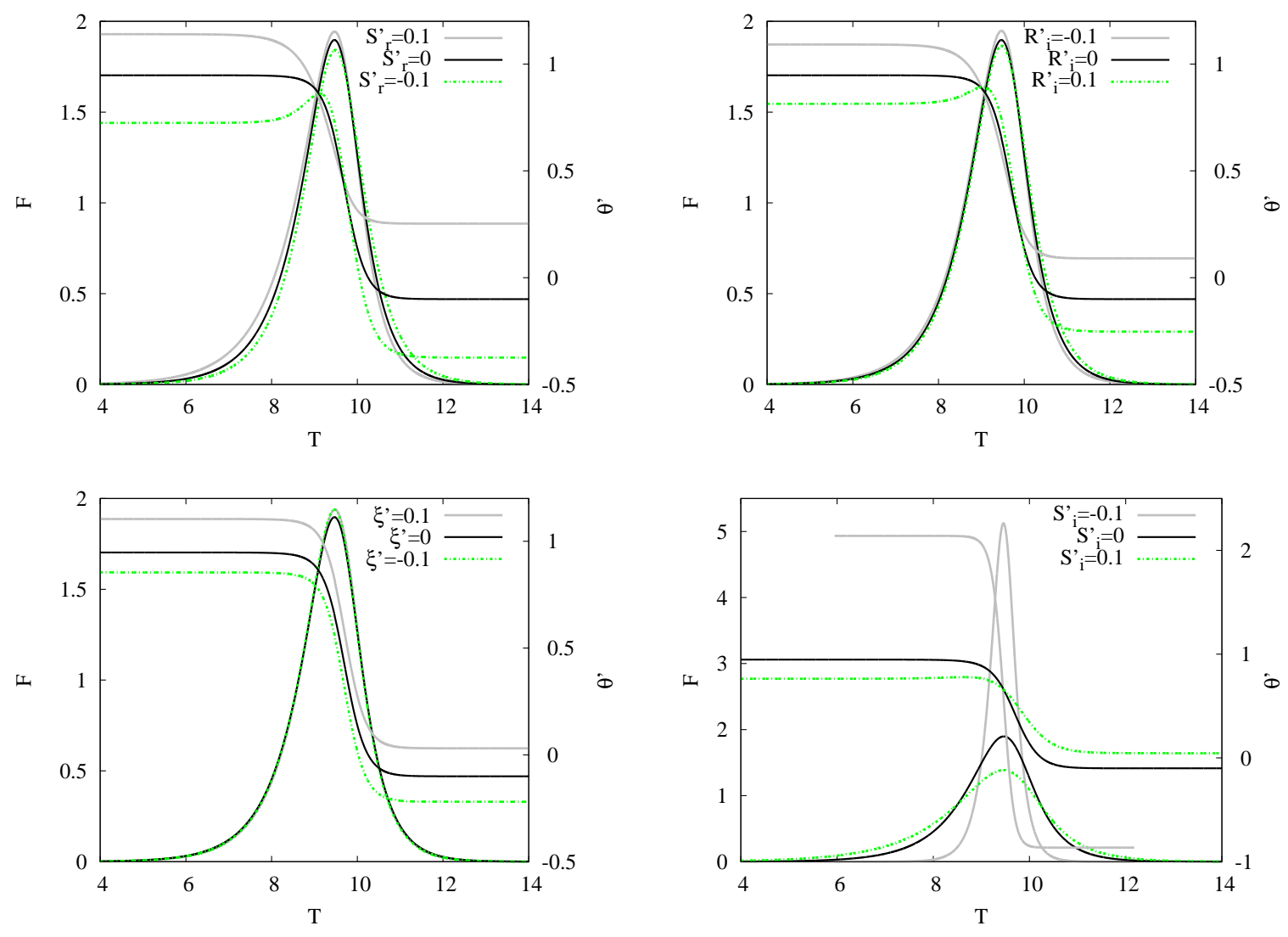

FIG. 4. Profiles of amplitude $(F)$ and derivative of phase $\left(\theta^{\prime}\right)$ for $\delta^{\prime}=-0.022, R_{r}^{\prime}=0.177, \beta=0.4$, $\epsilon=0.24$ and $S^{\prime}, R_{i}^{\prime}$ and $\xi^{\prime}$ as indicated in the labels.

in a future work.

We have also solved the full evolution equation (1) in order to confirm the Evans method results and to evaluate the basin of attraction of these stable solutions. The stability of the high amplitude solutions predicted by the Evans function method was confirmed by these simulations. Within the parameter region of stability, we have used input pulses of the form $A \operatorname{sech}(A t)$ with different $A$ s. These inputs stabilized to the stable pulse for a relatively large range of $A$ values as long as $\beta$ is not very low. Even for low $\beta$, and in the cases $R^{\prime}=0.177$ and $S^{\prime}=-0.1$ or $S^{\prime}=-0.1 \mathrm{i}$, it was possible to stabilize the propagation for different amplitudes of the sech. For larger values of $\beta$, the stabilization from the sech input was easier. The attraction basin for these solitons is especially large in the case $S^{\prime}=-0.1 \mathrm{i}$. We have analyzed three pairs of $\beta$ and $\epsilon: \beta=0.1$ and $\epsilon=0.15$ for which the $A \operatorname{sech}(A t)$ stabilized to the soliton for $A$ values ranging from 0.6 to $3.5, \beta=0.4$ and $\epsilon=0.2$ with stabilization for $A$ values from to 1.2 to 56.0 and $\beta=0.4$ and $\epsilon=0.32$ with stabilization from 0.6 
to 77.0. Whenever there was no stabilization, we observed pulse decay. Nevertheless, the stabilization was always possible if we use the profile obtained with the integration of the ODE.

\section{OSCILLATORY SOLITONS}

Thus far we have been discussing the existence and characteristics of the stable plain soliton solutions of equation (1). However, a very interesting behaviour is also governed by (1) as we enter the unstable region. In effect, by numerically integrating (1) we have observed the onset of stable oscillations as $\epsilon$ increases from the critical value associated with the upper boundary of the stable region. As we go further into the unstable region (increasing $\epsilon$ ), these oscillations increase in magnitude, such that the maximum pulse peak amplitude increases while the minimum one decreases. Moreover, the period of these oscillations also increases gradually, with the oscillations becoming further apart and eventually disappearing when the minimum peak amplitude reaches the amplitude of the low-amplitude stationary point possible in this region. Figure 5 depicts such behaviour when the HOT of the CGLE only includes the real Raman term. In the case represented in this figure, the oscillatory behaviour is only observed for a narrow range of $\epsilon$. However, as other terms are considered, this range can substantially increase. Furthermore, the amplitude of the oscillations can also increase considerably and, in some cases, we have even observed oscillations that were quite dramatic, which required special care with the simulation algorithm. An example of such behaviour, for a larger $\beta$ and also including $S_{r}$, is shown in Fig. 6. It is interesting to verify that in this case the oscillations persist for a much larger interval of $\epsilon$. Moreover, as $\epsilon$ increases, we observe that not only do the oscillations become larger and further apart, but they also become more asymmetric, with the descending edge varying quite abruptly. In figure 7, we show the oscillatory evolution of the pulse for the parameters as in Fig. 6 and $\epsilon=0.42$. Figure 8 shows, for the same case, the pulse energy, defined by $Q(Z)=\int_{-\infty}^{+\infty}|q|^{2} d T$, versus the peak amplitude which confirms the periodic character of this oscillations. In certain cases, we have observed a transition from periodic oscillations to non-periodic oscillations and again to periodic oscillations as $\epsilon$ is increased (see Fig. 9). In figure 10, we show the pulse energy versus peak amplitude for some $\epsilon$ of Fig. 9 that exhibit period doubling and period tripling. Also note that the oscillations depicted in figures 9 and 10 evolve very 


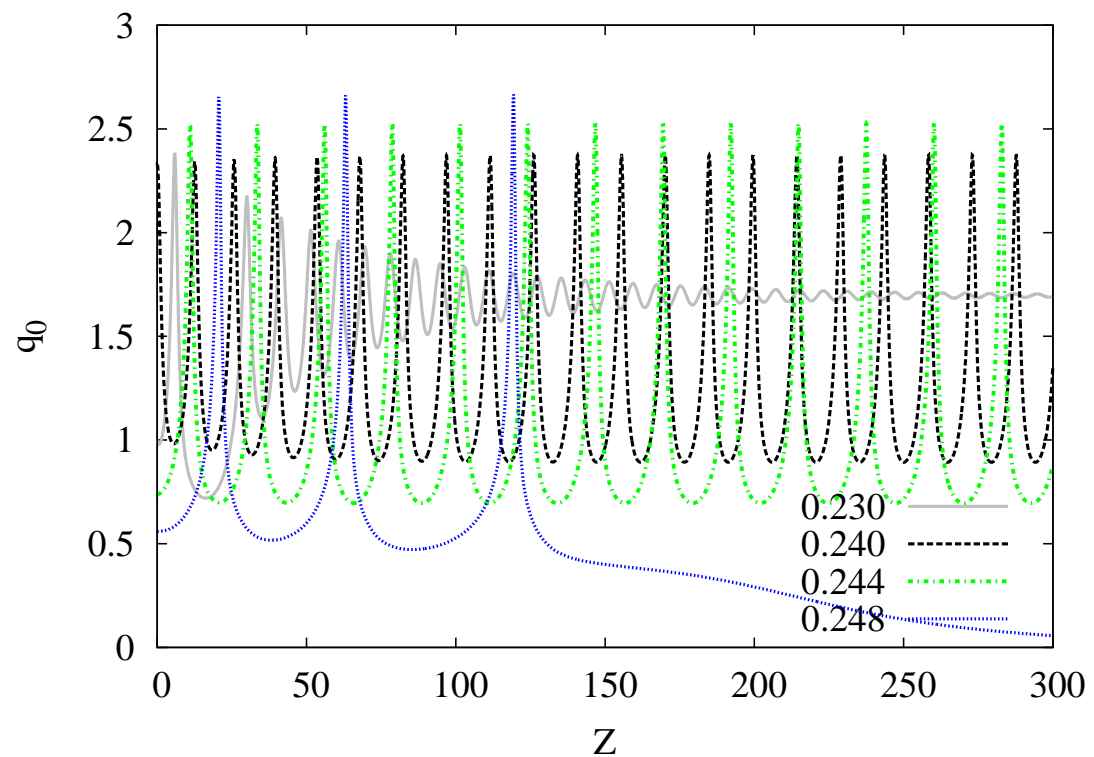

FIG. 5. Peak amplitude evolution for $\delta^{\prime}=-0.012, \beta=0.3, \xi^{\prime}=0, R^{\prime}=0.2, S^{\prime}=0$ and $\epsilon$ as in inset.

rapidly in the peak region and, moreover, that the difference between the maximum and the minimum peak amplitudes is quite large. For example, for $\epsilon=0.68$ we observe that within a period the minimum and maximum peak amplitudes are 1.6 and 74, respectively, whereas for $\epsilon=0.76$ this interval is larger $(0.6-93)$. This dramatic change becomes even more apparent when we compute the pulse energy in each case: for $\epsilon=0.68$ the minimum and maximum energies are respectively 3.9 and 538, while for $\epsilon=0.76$ we have 1.6 and 791 . In these last cases, we see that the pulse energy in a period changes more than two orders of magnitude.

It is noteworthy pointing out that a similar oscillatory behaviour was already known in the context of the cubic-quintic CGLE $[8,12-14]$ and for the cubic-quintic CGLE with higher-order terms $[10,11]$. However, to the best of our knowledge, this is the first time an oscillatory behaviour of this sort is predicted for the cubic CGLE.

\section{CONCLUSIONS}

We have presented results of existence and stability of plain solitons for the cubic CGLE with higher order terms included. The analysis shows that the cubic CGLE plus the Raman term is the minimal model to yield stable solitons. We know that, in the optics context, the 


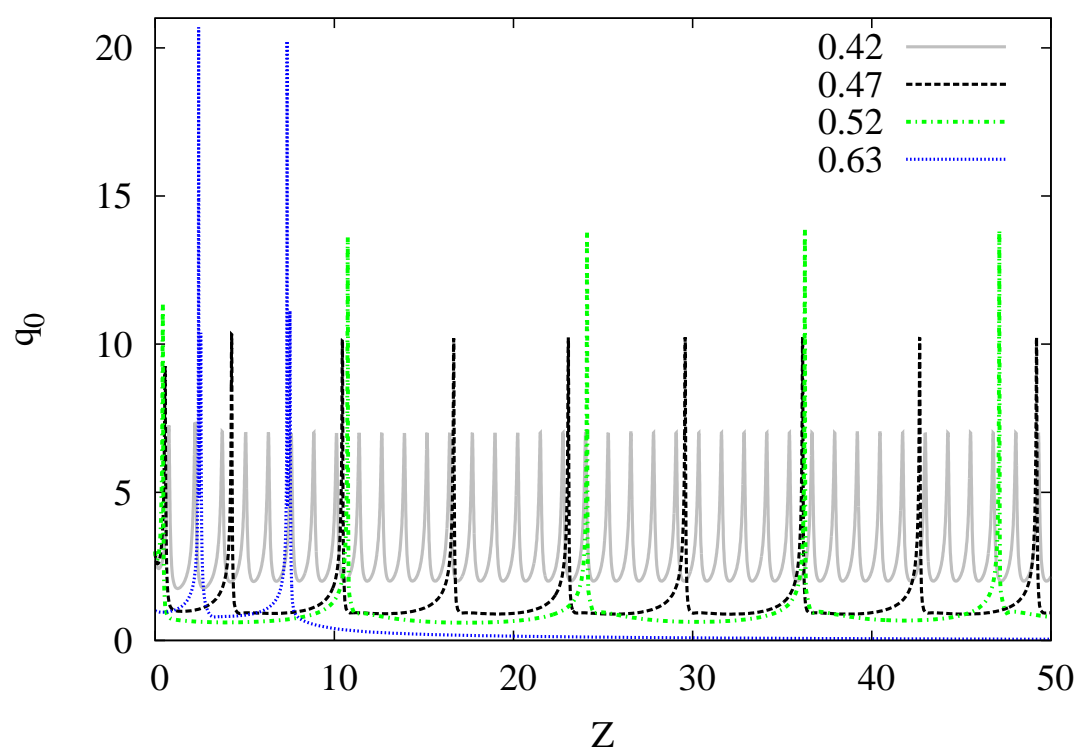

FIG. 6. Peak amplitude evolution for $\delta^{\prime}=-0.022, \beta=0.9, \xi^{\prime}=0, R^{\prime}=0.177, S^{\prime}=-0.1$ and $\epsilon$ as in inset.

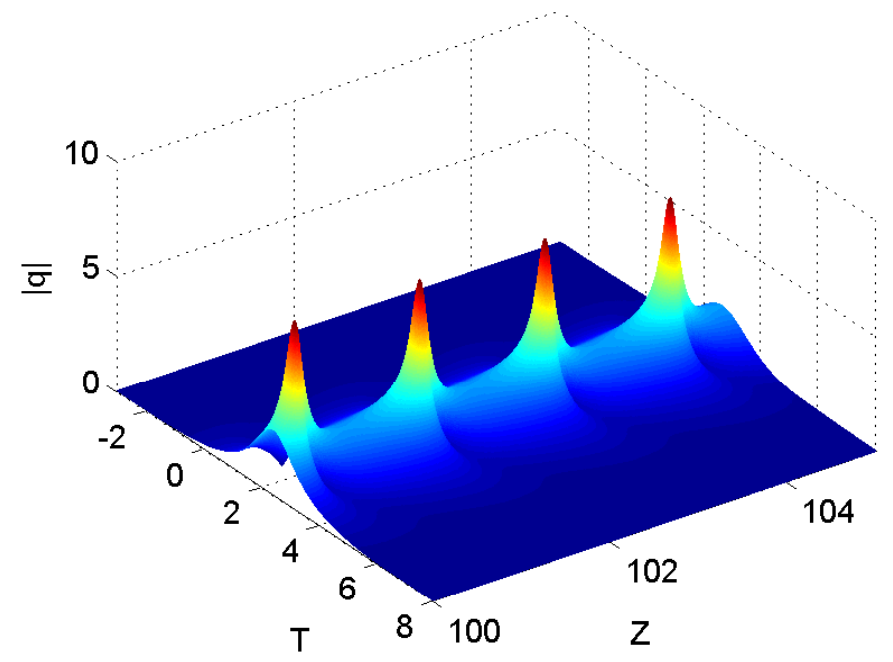

FIG. 7. Pulse evolution for the case $\epsilon=0.42$ of figure 6 .

Raman term is responsible for the continuous transfer of power from the blue to the red side of the spectrum and that the spectral filtering and the nonlinear gain would stabilize this process. We suppose that, in this case, this transference of power is somehow preventing the increase of amplitude that would occur for the same values of the cubic CGLE parameters. Nevertheless, the solution profile that allows this stabilization has higher amplitude. The existence of solitons is, in all analyzed cases, possible for a limited region of nonlinear gain 


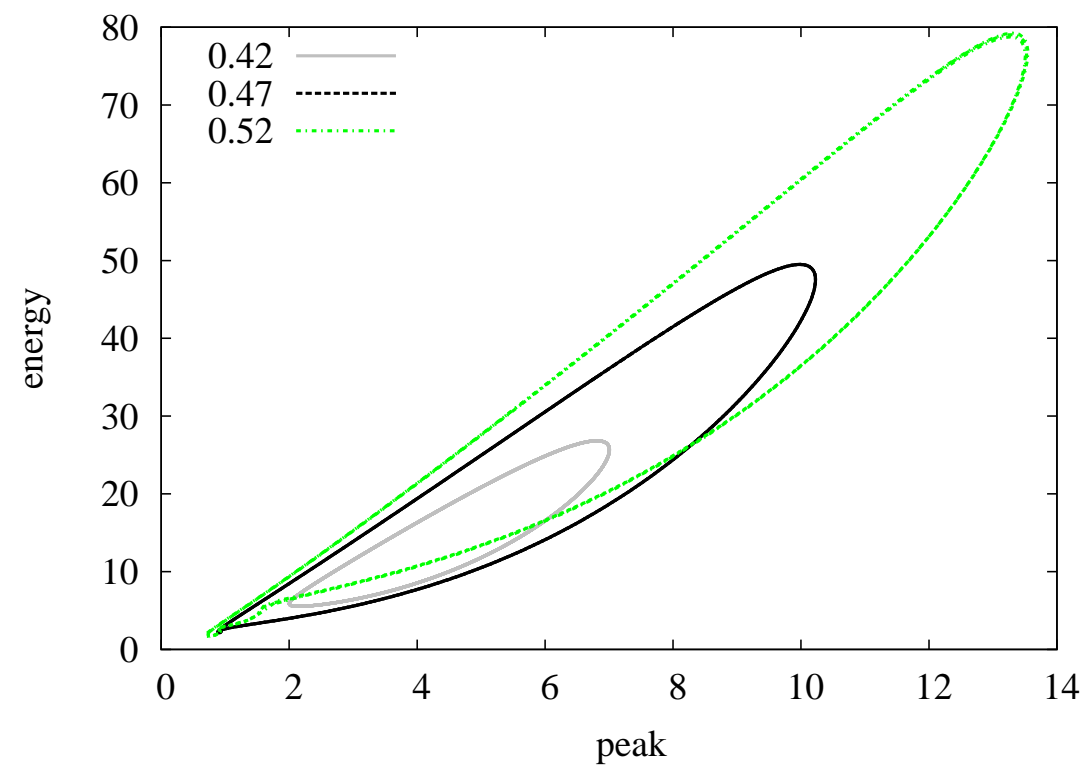

FIG. 8. Pulse energy versus peak amplitude for three of the cases represented in figure 6 as indicated in the inset.

and above a certain threshold of spectral filtering. The region of stability is particularly large for $S_{i}^{\prime}=-0.1$. The term associated with $S_{i}$ corresponds to a frequency dependence of the nonlinear gain and a negative value means that the nonlinear gain increases with frequency. The large region of stability and the large basin of attraction observed in this case is consistent with our above discussion; the Raman term is responsible for transference of power from higher to lower frequencies, the nonlinear gain participates in the compensation of this effect and a larger nonlinear gain on the high frequency side is beneficial. As we enter the unstable solitons region, the full integration of the evolution equation showed the existence of stable oscillatory solutions in all the studied cases. This transition corresponds to a bifurcation that will be further studied in a future work.

\section{ACKNOWLEDGMENTS}

The authors acknowledge Prof. Helmut Brand for helpful suggestions during the preparation of the manuscript. This work was partially financed by the ERDF - European Regional Development Fund through the Operational Programme for Competitiveness and Internationalisation - COMPETE 2020 Programme within project POCI-01-0145-FEDER-006961, and by National Funds through the FCT - Fundação para a Ciência e a Tecnologia (Por- 


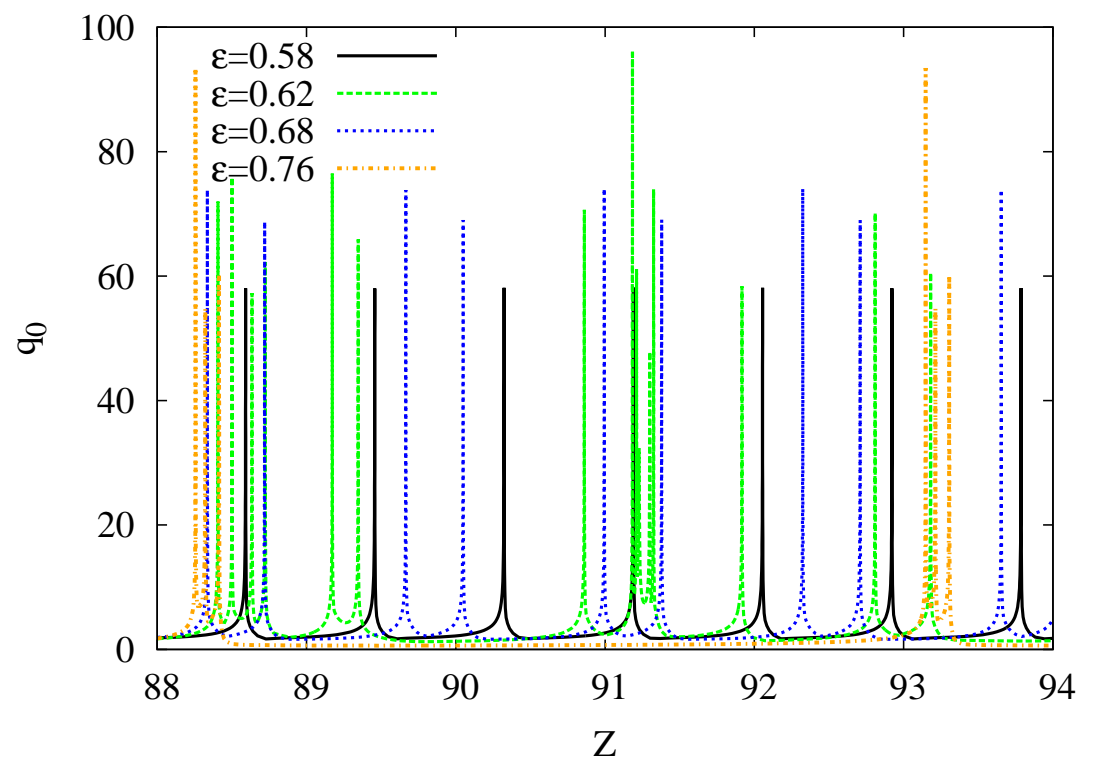

(a)

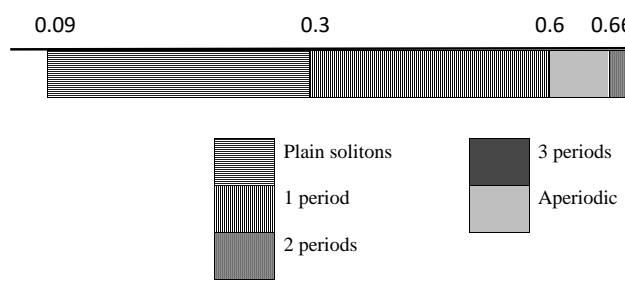

(b)

FIG. 9. (a) Peak amplitude evolution for $\delta^{\prime}=-0.022, \beta=0.1, \xi^{\prime}=0, R^{\prime}=0.177, S^{\prime}=-0.1 i$ and $\epsilon$ as in inset. (b) type of solutions across $\epsilon$ axis for the same parameters.

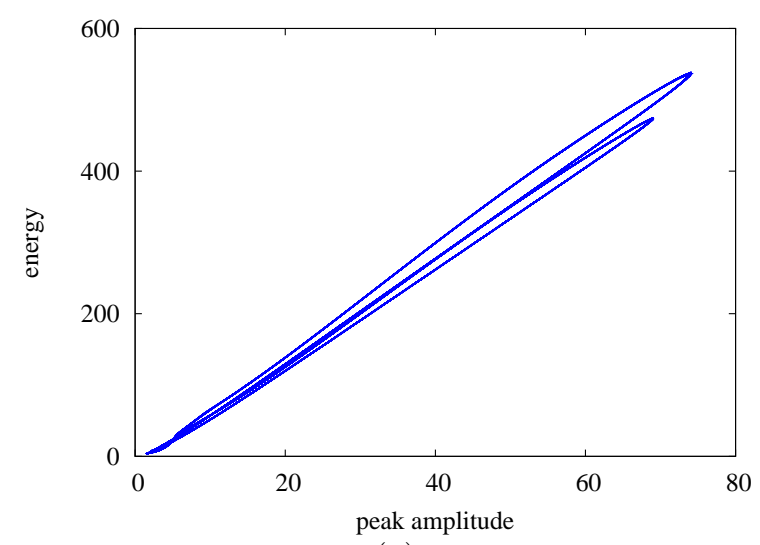

(a)

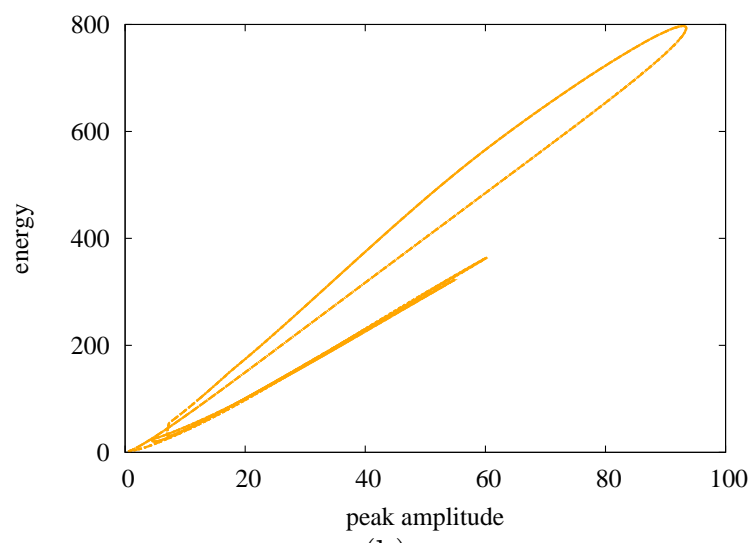

(b)

FIG. 10. Pulse energy versus peak amplitude for $\delta^{\prime}=-0.022, \beta=0.1, \epsilon=0.76, \xi^{\prime}=0, R^{\prime}=0.177$ and $S^{\prime}=-0.1 i$ and (a) $\epsilon=0.68$ and $(\mathrm{b}) \epsilon=0.76$. 
tuguese Foundation for Science and Technology) as part of projects UID/EEA/50014/2013 and UID/CTM/50025/2013.

[1] M. Matsumoto, H. Ikeda, T. Uda, and A. Hasegawa, J. Lightwave Technol. 13, 658 (1995).

[2] H. Haus, J. Fujimoto, and E. Ippen, J. Opt. Soc. Am. B 8, 2068 (1991).

[3] M. Facão, S. Rodrigues, and M. I. Carvalho, Phys. Rev. A 91, 013828 (2015).

[4] M. Facão and M. I. Carvalho, Phys. Rev. E 92, 022922 (2015).

[5] S. Latas and M. Ferreira, Opt. Lett. 35, 1771 (2010).

[6] M. Facão, M. I. Carvalho, S. C. Latas, and M. F. Ferreira, Phy. Lett. A 374, 4844 (2010).

[7] M. Facão and M. Carvalho, Physics Letters A 375, 2327 (2011).

[8] R. J. Deissler and H. R. Brand, Phys. Rev. Lett. 72, 478 (1994).

[9] N. Akhmediev, J. M. Soto-Crespo, and G. Town, Phys. Rev. E 63, 056602 (2001).

[10] R. J. Deissler and H. R. Brand, Phys. Rev. Lett. 81, 3856 (1998).

[11] C. Cartes and O. Descalzi, Philosophical Transactions of the Royal Society A - Mathematical Physical and Engineering Sciences 373, 20150114 (2015).

[12] E. Tsoy and N. Akhmediev, Phys. Lett. A 343, 417 (2005).

[13] E. Tsoy, A. Ankiewicz, and N. Akhmediev, Phys. Rev. E 73, 036621 (2006).

[14] W. Chang, J. Soto-Crespo, P. Vouzas, and N. Akhmediev, Phys. Rev. E 92, 022926 (2015).

[15] G. Huang, L. Deng, and M. G. Payne, Phys. Rev. E 72, 016617 (2005).

[16] R. J. Deissler and H. R. Brand, Physics Letters A 146, 252 (1990).

[17] D. J. Kaup and A. C. Newell, Proceedings of the Royal Society of London. A. Mathematical and Physical Sciences 361, 413 (1978).

[18] V. I. Karpman, Physica Scripta 20, 462 (1979).

[19] N. Akhmediev, V. Afanasjev, and J. Soto-Crespo, Phys. Rev. E 53, 1190 (1996).

[20] D. Henry, Geometric theory of semilinear parabolic equations, 3rd ed., Lecture Notes in Mathematics, Vol. 840 (Springer-Verlag, Berlin, 1993). 